\title{
Development of New Left Atrial Anatomical Models For the Study of the Left Atrial Appendage Implications in Atrial Fibrillation
}

\author{
Alessandro Masci ${ }^{1}$, Lorenzo Barone ${ }^{1}$, Francesca Mazzotti $^{1}$, Corrado Tomasi $^{2}$, Cristiana Corsi $^{1}$ \\ ${ }^{1}$ DEI, University of Bologna, Campus of Cesena, Bologna, Italy \\ ${ }^{2}$ Deparment of Cardiology, Ospedale Santa Maria delle Croci, Ravenna, Italy
}

\begin{abstract}
Atrial fibrillation is associated with a five-fold increase of the stroke risk. Left atrial appendage (LAA) is the atrial site with the highest blood stasis risk, increasing thrombus formation and stroke. Recent studies have been focused on the association between the left atrial appendage anatomical features and the stroke risk. However, conflicting results have been published. In this context, clinical studies suggested the stroke risk stratification could be improved by using haemodynamics information on the left atrium and mainly on the left atrial appendage. Therefore, the aim of this study was the design and development of a method which enabled to reconstruct and generate several LA anatomical models, where each one was characterized by a different LAA morphology. These anatomical models represent the computational domain for the computational fluid dynamics simulations of the haemodynamics within the left atrium and LAA.
\end{abstract}

\section{Introduction}

In order to prevent the stroke risk, warfarin was the only option available until recently. However, the administration of warfarin has many limitations, including constant monitoring, inter-individual variability in anticoagulant effect, food and drug interactions, etc. [3]. For these reasons, different strategies have been developed such as the use of left atrial appendage (LAA) occlusion devices, which seem to better reduce the risk of thromboembolism compared to warfarin anticoagulation therapy [4]. LAA is the remnant of the embryonic left atrium (LA), whereas the smooth-walled left atrium originates from the primordial pulmonary vein and its branches. Because of its hooked morphology, LAA is the left atrial site with the highest blood stasis risk, increasing thrombus formation and stroke. In fact, $90 \%$ of the intracardiac thrombi in patients with cardioembolic stroke/TIA originally develop in the LAA [5]. Therefore, morphological and quantitative features of LAA have been increasingly studied in order to improve the knowledge on the LAA implications in AF. Di Biase et al [6] presented the classification of LAA morphology into four different classes (chicken wing, cauliflower, windsock and cactus, see Figure 1) and studied the correlation between this characteristic and the patient-specific stroke history. Results demonstrated that chicken wing LAA morphology, which is the most prevalent, was less likely to have an embolic event compared to the other LAA types. Yet, Jeong et al [7] determined
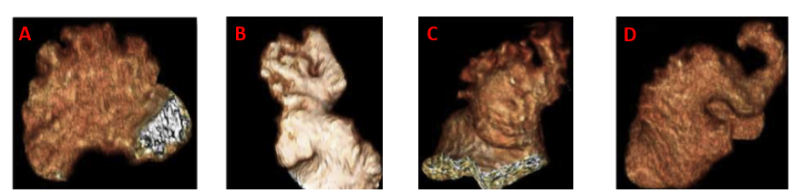

Figure 1. Classification of the LAA different morphologies: Cauliflower(A), Windsock(B), Chicken Wing(C), Cactus (D).

whether morphometric or volumetric parameters of LAA would be related to the development of cardioembolism in subjects with AF. They found that LAA orifice diameter and LAA volume were larger in patients with a history of cardioembolic stroke with respect to a control group. AF implies alterations in the LAA wall and surrounding structures and this offers a possible pathophysiological explanation for elevated LAA volumes in stroke patients. Despite these attempts, the association between the aforementioned LAA anatomical features and stroke risk has not been deeply investigated, and conflicting results have been published. Moreover, it is still uncertain what is the best strategy for stroke prevention in AF. To this purpose, several clinical studies suggested that stroke risk stratification could be improved by using hemodynamic information on the left atrium (LA) and mainly on the LAA. Computational fluid dynamics (CFD) represents a valuable non-invasive tool to determine and assess physically meaningful parameters and indicators in a complex fluid dynamics system, such as velocity, the cardiac blood flowrates, vorticity, turbulent kinetic energy, etc. Therefore, the aim 


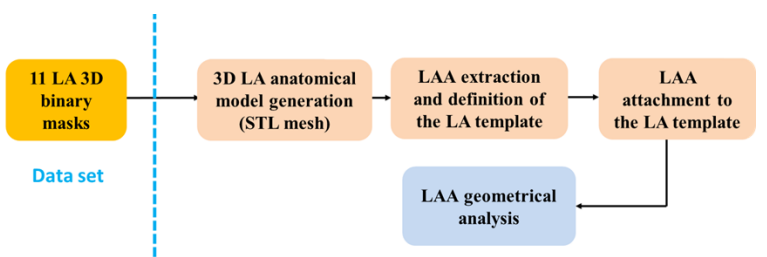

Figure 2. A schematic description of the workflow of this study.

of this study was the design and development of a workflow which enabled to reconstruct and generate several LA anatomical models, where each one was characterized by the same LA chamber and a different LAA morphology . These anatomical models will represent the computational domain for the CFD model described in [8] and simulations of the haemodynamics within the LA and LAA will be performed in the next future. In this paper we discuss the details of the developed pipeline, starting from a data-set of 3D LA (including LAA) anatomical models, obtained by real clinical data (CT and MRI). A quantitative analysis for each LAAwas performed to evaluate the most significative geometrical parameters, such as the volume, the surface, the number of lobes and the LAA orifice.

\section{Methods}

A schematic workflow of the developed analysis process is shown in Figure 2.

\subsection{Patients data}

Data-set consisted in eleven LA 3D anatomical models, extracted from CT and MRI data with specifically image segmentation algorithms, described in $[8,9]$. From the 3D LA binary masks, we generated the surface meshes by using the MATLAB iso2mesh toolbox [10].

\subsection{LAA extraction}

Next step was focused on the implementation of an algorithm which automatically recognized and isolated the LAA. To this purpose, the shape diameter function (SDF) proposed by Shapira et al. [11] was employed. In this study, the SDF was calculated for each 3D surface using the CGAL software. An example of the SDF values computation for one LA anatomical model is depicted in Figure 3. Iso-contours of the SDF map on the LA mesh and was used to separate regions with different SDF values. Based on this idea, we obtained a semantic clusterization of our 3D models in which each cluster had a different id number. The output of this procedure was a vector that contained the id number for each facet. This step allowed us to identify pulmonary veins (PVs), LA chamber and LAA

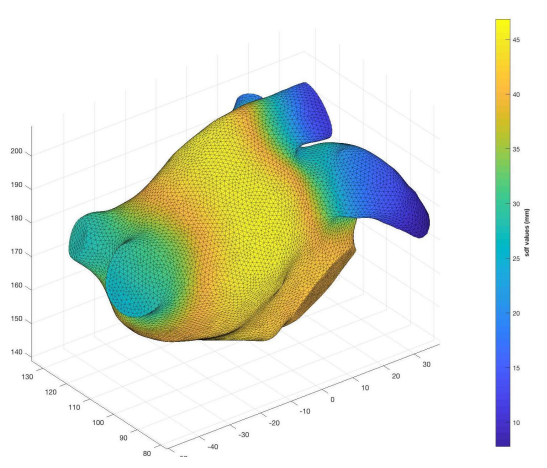

Figure 3. An example of the SDF result on a LA mesh.

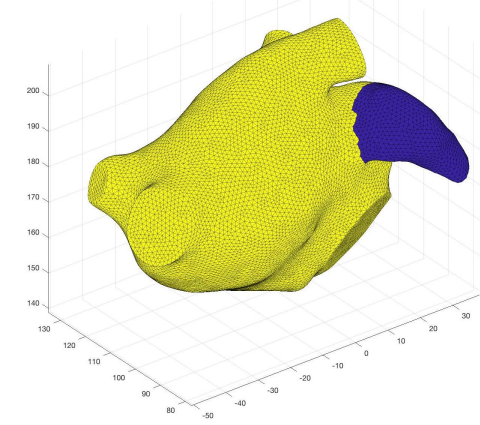

Figure 4. LAA vertices and faces detection.

for each mesh. In order to improve the LAA identification, we detected the atrial chamber id by calculating the mode (statistics) of the id vector; afterwards we assigned to the PVs the same id of the LA chamber. Based on the anatomical position of the LAA we were able to select this region of interest in order to automatically identify it and isolate from the LA surface mesh. An example of the result of this step is shown in Figure 4.

Therefore, we obtained eleven LAA surface meshes. Once the LAA was removed from each LA model, we selected one model as LA template. This specific model was used as template for the definition of the new eleven LA anatomical models as described in the next section.

\subsection{LAA alignment and definition of the new LA models}

Once performed the previous steps, in order to accomplish the alignment between the LA template and the LAA meshes, we identified the vertices which belong to LAA ostia and to the ostium of the LA template. The output was a vector that contained the coordinates of these detected vertices. The local correspondence between LAA and LA template ostium vertices was achieved by applyiing the it- 

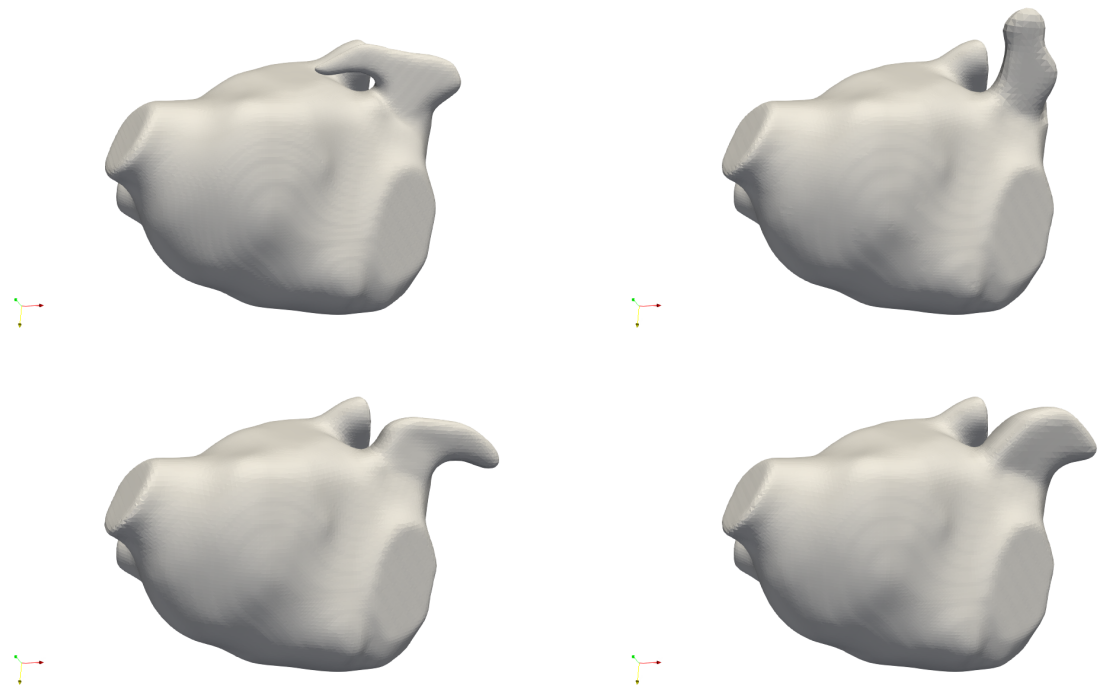

Figure 5. Example of four LA anatomical models obtained by the automatic framework previously described.

erative closest point (ICP) algorithm [12]. The ICP algorithm matches closest points between two point data sets: one was used as a fixed data set and the other as a floating data set. In our case, the fixed one was composed by vertices of the LA ostium and the floating one by the LAA ostium vertices.

Finally, in order to refine the anatomical models and to comply with the requirement of providing a smooth geometrical representation of the computational domain for the CFD simulation, a Laplacian smoothing filter and a Poisson surface reconstruction were applied by using MeshLab [13]. Once this procedure was completed, a set of eleven LA anatomical models, presenting the same atrial chamber but different LAA morphologies and geometries, was created.

\section{Results and Discussion}

Four of the eleven anatomical models obtained applying this procedure are illustrated in Figure 5. From the figure, we notice that the shape and the geometry of the LAAs are very different and their morphologies are in accordance with the classification proposed by Di Biase et al. [6].

Furthermore, in order to quantify the differences between the LAAs, we performed a specific geometrical analysis by quantifying volume, surface area, length, turtuosity and ostium area of each LAA.

Length was evaluated by the LAA centerlines computation.

Tortuosity was defined as:

$$
\mathcal{X}=\frac{\mathcal{L}}{\mathcal{D}} ;
$$

where $\mathrm{L}$ is the centerline length and $\mathrm{D}$ is the Euclidean distance between the endpoints. Tortuosity values higher than 1 reflected the complexity of the LAA shapes, whereas values near to 1 meant that LAA geometry was more linear. In order to compute the LAA volumes and orifice (i.e. LAA ostium) areas, LAA volume meshes were generated. The values of these parameters are reported in Table 1. LAA volume presented high variability between the analyzed patients and it could be related to the stage or degree of AF disease progression. However, this clinical information was not available for the patients considered in this study. After this careful analysis, the next step will be focused on the haemodynamics simulation of each anatomical models in order to study the effects of each geometrical feature on the blood flow pattern within LAA.

\section{Conclusions}

In this work we described in detail an automatic framework which enabled to reconstruct and generate several LA anatomical models, where each one was characterized by a different LAA morphology and shape. We also characterized the morphology of the LAAs by computing several geometrical parameters able to characterize their shapes. Simulations of the computational fluid dynamics within these new LA anatomical model, focusing in particular on the LAA, will be employed in the next future in order to investigate the correlation between the haemodynamic parameters (i.e. velocity, vorticity, kinetic energy, residence time etc.) and the LAA geometrical characteristics.

The CFD simulation could improve the knowledge on intra-atrial blood stasis and on the probability of clot for- 


\begin{tabular}{cccccc} 
LAA & Volume $\left(\mathrm{cm}^{3}\right)$ & Surface area $\left(\mathrm{cm}^{2}\right)$ & Ostium area $\left(\mathrm{cm}^{2}\right)$ & Length $(\mathrm{cm})$ & Tortuosity \\
\hline 1 & 3.97 & 12.03 & 2.64 & 2.85 & 1.15 \\
2 & 2.15 & 7.94 & 1.77 & 1.87 & 1.06 \\
3 & 2.31 & 9.39 & 1.86 & 3.62 & 1.38 \\
4 & 3.60 & 11.08 & 2.47 & 2.54 & 1.03 \\
5 & 1.96 & 6.71 & 1.91 & 1.70 & 1.04 \\
6 & 2.12 & 8.31 & 1.15 & 2.31 & 1.20 \\
7 & 1.23 & 5.45 & 1.38 & 1.65 & 1.09 \\
8 & 2.60 & 10.10 & 1.96 & 2.82 & 1.12 \\
9 & 3.35 & 10.09 & 2.60 & 2.31 & 1.12 \\
10 & 9.06 & 20.78 & 4.51 & 2.92 & 1.03 \\
11 & 2.21 & 9.11 & 1.77 & 2.52 & 1.05 \\
\hline
\end{tabular}

Table 1. LAA geometrical parameters

mation within the different LAAs and potentially enables optimized patient risk stratification and therapy.

\section{References}

[1] Zakeri R, Van Wagoner DR, Calkins H, Wong T, Ross HM, Heist EK, Meyer TE, Kowey PR, Mentz RJ, Cleland JG, et al. The Burden of Proof: the Current State of Atrial Fibrillation prevention and treatment trials. Heart Rhythm 2017;14(5):763-782.

[2] With the special contribution of the European Heart Rhythm Association (EHRA) D, by the European Association for Cardio-Thoracic Surgery (EACTS) E, Members AF, Camm AJ, Kirchhof P, Lip GY, Schotten U, Savelieva I, Ernst S, Van Gelder IC, et al. Guidelines For the Management of Atrial Fibrillation: the Task Force For the Management of Atrial Fibrillation of the European Society of Cardiology (ESC). European heart journal 2010;31(19):23692429.

[3] Hankey GJ, Eikelboom JW. Dabigatran Etexilate: a New Oral Thrombin Inhibitor. Circulation 2011;123(13):14361450.

[4] Reddy VY, Möbius-Winkler S, Miller MA, Neuzil P, Schuler G, Wiebe J, Sick P, Sievert H. Left Atrial Appendage Closure with the Watchman Device in Patient with a Contraindication For Oral Anticoagulation: the ASAP Study (asa plavix feasibility study with watchman left atrial appendage closure technology). Journal of the American College of Cardiology 2013;61(25):2551-2556.

[5] Yaghi S, Song C, Gray WA, Furie KL, Elkind MS, Kamel H. Left Atrial Appendage Function and Stroke Risk. Stroke 2015;46(12):3554-3559.

[6] Di Biase L, Santangeli P, Anselmino M, Mohanty P, Salvetti I, Gili S, Horton R, Sanchez JE, Bai R, Mohanty S, et al. Does the Left Atrial Appendage Morphology Correlate with the Risk of Stroke in Patients with Atrial Fibril- lation?: Results From a Multicenter Study. Journal of the American College of Cardiology 2012;60(6):531-538.

[7] Jeong WK, Choi JH, Son JP, Lee S, Lee MJ, Choe YH, Bang OY. Volume and Morphology of Left Atrial Appendage as Determinants of Stroke Subtype in Patients with Atrial Fibrillation. Heart Rhythm 2016;13(4):820-827.

[8] Masci A, Alessandrini M, Forti D, Menghini F, Dedé L, Tommasi C, Quarteroni A, Corsi C. A Patient-specific Computational Fluid Dynamics Model of the Left Atrium in Atrial Fibrillation: Development and Initial Evaluation. In International Conference on Functional Imaging and Modeling of the Heart. Springer, 2017; 392-400.

[9] Valinoti M, Fabbri C, Turco D, Mantovan R, Pasini A, Corsi C. 3D Patient-specific Models for Left Atrium Characterization to Support Ablation in Atrial Fibrillation Patients. Magnetic resonance imaging 2018;45:51-57.

[10] Fang Q, Boas D. Iso2mesh: a 3D Surface and Volumetric Mesh Generator for Matlab/Octave, 2010.

[11] Shapira L, Shamir A, Cohen-Or D. Consistent Mesh Partitioning and Skeletonisation Using The Shape Diameter Function. The Visual Computer 2008;24(4):249.

[12] Besl PJ, McKay ND. Method for Registration of 3D Shapes. In Sensor Fusion IV: Control Paradigms and Data Structures, volume 1611. International Society for Optics and Photonics, 1992; 586-607.

[13] Cignoni P, Callieri M, Corsini M, Dellepiane M, Ganovelli F, Ranzuglia G. Meshlab: an Open-source Mesh Processing Tool. In Eurographics Italian Chapter Conference, volume 2008. 2008; 129-136.

Address for correspondence:

Cristiana Corsi, $\mathrm{PhD}$

DEI, University of Bologna

Via Risorgimento 2, 40136 Bologna, Italy

E-mail address: cristiana.corsi3@unibo.it 